\title{
Issues in Underwater Acoustic Sensor Networks
}

\author{
Manjula.R.B, Sunilkumar S. Manvi
}

\begin{abstract}
The oceans remain the least explored frontiers on this planet and many oceanic and maritime applications seem relatively slow in exploiting the state-of-the-art info-communication technologies. The natural and man-made disasters that have taken place over the last few years have aroused significant interest in monitoring oceanic environments for scientific, environmental, commercial, safety, homeland security and military needs. The shipbuilding and offshore engineering industries are also increasingly interested in technologies like sensor networks as an economically viable alternative to currently adopted and costly methods used in seismic monitoring, structural health monitoring, installation and mooring, etc. Underwater sensor networks (UWSNs) are the enabling technology for wide range of applications like monitoring the strong influences and impact of climate regulation, nutrient production, oil retrieval and transportation

The underwater environment differs from the terrestrial radio environment both in terms of its energy costs and channel propagation phenomena. The underwater channel is characterized by long propagation times and frequency-dependent attenuation that is highly affected by the distance between nodes as well as by the link orientation. Some of other issues in which UWSNs differ from terrestrial are limited bandwidth, constrained battery power, more failure of sensors because of fouling and corrosion, etc. This paper presents several fundamental key aspects and architectures of UWSNs, emerging research issues of underwater sensor networks and exposes the researchers into networking of underwater communication devices for exciting ocean monitoring and exploration applications.
\end{abstract}

Index Terms - Underwater Sensor Networks (UWSNs), Acoustic Communication, Distance Aware Collision Avoidance (DACAP)

\section{INTRODUCTION}

The Earth is a water planet. Around $70 \%$ of the surface of earth is covered by water. This is largely unexplored area and recently it has fascinated humans to explore it. Natural or man-made disasters that have taken place over the last few years have aroused significant interest in monitoring oceanic environments for scientific, environmental, commercial, safety, homeland security and military needs. The shipbuilding and offshore engineering industries are also increasingly interested in technologies like wireless sensor

Manuscript received June 23, 2010.

Manjula.R.B and Sunilkumar Manvi are with the Department of Electronics and Communication Engineering. Reva Institute of Technology and Management, Bangalore-560064, India. (Phone number: +91-80-65687563)

e-mail:\{manjula@revainstitution.org,sunil.manvi@revainstitution.org\} networks as an economically viable alternative to currently adopted and costly methods used in seismic monitoring, structural health monitoring, installation and mooring, etc. There has been a growing interest in monitoring underwater mediums for scientific exploration, commercial exploitation, and attack protection as it contributes for human well being.

Major technological advances in related fields have opened the horizon for numerous novel networking schemes. Sensor networks have revolutionized all the areas of technology i.e. science, industry and government. The revolution is due to the miniaturization and the advancement in technology i.e. availability of low powered processing, storage units and Micro Electrical and Mechanical Systems (MEMS) for constructing onboard sensing units. The ability to have small devices physically distributed near the objects being sensed brings new opportunities to observe and act on the world, for example with micro-habitat monitoring [1] [2], structural monitoring [3], and industrial applications [4]. While sensor-net systems are beginning to be fielded in applications today on the ground, underwater operations remain quite limited by comparison.

Wireless underwater networking is the enabling technology for ocean applications. Under water sensor network consists of a variable number of sensors and vehicles that are deployed to perform collaborative monitoring tasks over a given area. To achieve this objective, sensors and vehicles self-organize in an autonomous network which can adapt to the characteristics of the ocean environment. Underwater networks can be characterized by their spatial coverage and by the density of nodes.

Underwater sensor networks mainly differ in the communication media employed for information transmission. The work given in [56] reviews the physical fundamentals and engineering implementations for efficient information exchange via wireless communication using physical waves as the carrier among nodes in an underwater sensor network. The physical waves include sound, radio, and light. Based on the comparison study, one can select carriers for underwater sensor networks that enhance the communication efficiency in specified underwater environment. The major characteristics of acoustic, electromagnetic and optical carriers are given in Table 1.

\section{A. Differences between underwater sensor networks and terrestrial networks}

Underwater sensor networks are quite different from terrestrial sensor networks [10]. The differences between these are as follows. 
Comparison of Acoustic, EM and Optical Waves In Sea Environment

\begin{tabular}{|l|l|l|l|}
\hline \multicolumn{1}{|c|}{ Parameter } & \multicolumn{1}{|c|}{ Acoustic } & \multicolumn{1}{c|}{ EM } & \multicolumn{1}{c|}{ Optical } \\
\hline Nominal speed & $\sim 1500$ & $\sim 33,333,333$ & $\sim 33,333,333$ \\
\hline Power loss & $>0.1 \mathrm{db} / \mathrm{m} / \mathrm{Hz}$ & $\sim 28 \mathrm{db} / 1 \mathrm{~km} / 100 \mathrm{MHz}$ & Propto turbidity \\
\hline Bandwidth & $\sim \mathrm{KHz}$ & $\sim \mathrm{MHz}$ & $\sim 10-150 \mathrm{MHz}$ \\
\hline Frequency band & $\sim \mathrm{KHz}$ & $\sim \mathrm{MHz}$ & $\sim 10^{14}-10^{13} \mathrm{~Hz}$ \\
\hline Antenna size & $\sim 0.1 \mathrm{~m}$ & $\sim 0.5 \mathrm{~m}$ & $\sim 0.1 \mathrm{~m}$ \\
\hline Effective range & $\sim \mathrm{km}$ & $\sim 10 \mathrm{~m}$ & $\sim 10-100 \mathrm{~m}$ \\
\hline
\end{tabular}

Table 1

- Communication method: Terrestrial sensor networks employ electromagnetic waves but in underwater networks because of the characteristic (large delay, long distance of communication) of network, the communication is relied on physical means like acoustic sounds to transmit the signal. Traditional RF networks might not work efficiently in underwater networks.

- Protocols: Due to distinct network dynamics, existing communication protocols for terrestrial networks are not suitable for underwater environment. Low bandwidth and large latency result in long end to end delays, and this brings in challenges in reliable data transfer and traffic congestion control.

- Cost: Terrestrial networks are becoming inexpensive due to advancement in technology but underwater sensors are still expensive devices. This is due to the extra protection required for underwater environment and more complex transceivers needed.

- Deployment: Terrestrial networks are densely deployed, whereas in underwater networks the deployment is sparser due to the challenges involved in deployment and also the cost involved in deployment.

- Power: In terrestrial networks, due to higher distance of communication, type of media being used for communication, signal processing techniques involved at the transceivers, the power required is more and complex as compared to terrestrial sensor networks.

- Node Mobility: In case of terrestrial networks nodes mobility can be predicted whereas in the underwater networks prediction of mobility of the node is difficult, because of the density and flow variation of the water.

- Memory: Underwater sensors need to have large memory compared to terrestrial sensors as the underwater channel is intermittent.

- Spatial Correlation: Readings taken from terrestrial networks with sensors are often correlated but this is not the case in underwater networks as the distance is more among the sensors.

\section{B. Unique characteristics of underwater acoustic sensor networks}

- Communication media: Underwater communication system involves transmission of information using any media either acoustic waves, electromagnetic waves or optical waves. Each of the techniques have their own advantages and limitations as discussed in Section 3.1. Acoustic communication is the most versatile and widely used technique in underwater due to low attenuation in water.

- Transmission loss: It consists of attenuation and geometric spreading. The attenuation is mainly provoked by absorption due to conversion of acoustic energy into heat, and increases with distance and frequency. The geometric spreading refers to the spreading of sound energy as a result of the expansion of the wavefronts. It increases with the propagation distance and is independent of frequency.

- Noise: It can be classified as man-made noise and ambient noise. The former is mainly caused by machinery noise (pumps, reduction gears, power plants), and shipping activity (hull fouling, animal life on hull, cavitation), while the latter is related to hydrodynamics (movement of water including tides, current, storms, wind, and rain), and to seismic and biological phenomena.

- Multipath: Multipath propagation may be responsible for severe degradation of the acoustic communication signal, since it generates Inter Symbol Interference (ISI). The multipath geometry depends on the link configuration. Vertical channels are characterized by little time dispersion, whereas horizontal channels may have long multipath spreads. The extent of the spreading is a strong function of depth and the distance between the transmitter and the receiver.

- Doppler spread: The Doppler frequency spread causes degradation in the performance of digital communications. The Doppler spreading generates two effects: a simple frequency translation and a continuous spreading of frequencies, which constitutes a non-shifted signal. While the former is easily compensated at the receiver, the effect of the latter is harder to be compensated for.

\section{Challenges in underwater acoustic sensor networks}

Major challenges encountered in the design of underwater acoustic networks are as follows [10] [12].

1 The available bandwidth is severely limited.

2 The underwater channel is impaired because of multi-path and fading.

3 Propagation delay in underwater is five orders of magnitude higher than in Radio Frequency (RF) terrestrial channels, and variable.

4 High bit error rates and temporary losses of connectivity (shadow zones) can be experienced.

5 Underwater sensors are characterized by high cost because of extra protective sheaths needed for sensors and also relatively small number of suppliers (i.e., not much economy of scale) are available.

6 Battery power is limited and usually batteries cannot be recharged as solar energy cannot be exploited.

7 Underwater sensors are more prone to failures because of fouling and corrosion. 


\section{Applications of underwater sensor networks}

The application of wireless sensor networks to the underwater domain has huge potential for monitoring the health of river and marine environments [11]. Monitoring these environments is difficult and costly for humans: divers are regulated in the hours and depths at which they can work, and require a boat on the surface that is costly to operate and subject to weather conditions. A sensor network deployed underwater could monitor physical variables such as water temperature and pressure as well as variables such as conductivity, turbidity and certain pollutants. The network could track plumes of silt due to dredging operations or pollutants owing in from land, and it could monitor and model the behavior of underwater ecosystems. Imaging sensors could be used to measure visible change in the environment or count, and perhaps even classify species and also useful for disaster prevention. The applications of underwater sensor networks are broadly classified as follows [10].

1) Long term non-time critical applications: In UWSN architecture for long-term non-time-critical aquatic monitoring applications, sensor nodes are densely deployed to cover a spatial continuous monitoring area. Data are collected by local sensors and relayed by intermediate sensors, and then finally reach the surface nodes (equipped with both acoustic and RF modems), which can transmit data to the on-shore command center by radio. The applications concerned to long term non-time critical applications are as follows.

- Ocean sampling networks: Networks of sensors and underwater vehicles can perform synoptic, cooperative adaptive sampling of the coastal ocean environment [5] [6].

- Environmental monitoring: Underwater acoustic sensor networks (UW-ASNs) can perform pollution monitoring (chemical, biological and nuclear) [7]. For example, it may be possible to detail the chemical slurry of antibiotics, estrogen-type hormones and insecticides to monitor streams, rivers, lakes and ocean bays (water quality in situ analysis). Monitoring of ocean currents and winds, improved weather forecast, detecting climate change, under-standing and predicting the effect of human activities on marine ecosystems, biological monitoring such as tracking of fishes or micro-organisms, are other possible applications.

- Undersea explorations: Underwater sensor networks can help to detect underwater oilfields or reservoirs, determine routes for laying undersea cables, and assist in exploration for valuable minerals.

- Flocks of Underwater Robots: A very different application is supporting groups of underwater autonomous robots. Applications include coordinating adaptive sensing of chemical leaks or biological phenomena (for example, oil leaks or phytoplankton concentrations), and also equipment monitoring applications as described above.

- Mine reconnaissance: The simultaneous operation of multiple underwater acoustic vehicles with acoustic and optical sensors can be used to perform rapid environmental assessment and detect mine-like objects [9].
2) Short-term time critical acoustic exploration: The applications which are of short term, and time of response plays a vital role are included in this category [8].

- Civilian Scenario: One of the important applications of the mobile UWSN architecture for short-term time-critical aquatic exploration applications is the civilian scenario. Assume a ship-wreckage and the accident-investigation team wants to identify the target venue. Existing approaches usually employ tethered wire/cable to a remotely operated vehicle (ROV). When the cable is damaged, the ROV is out of control or not recoverable. In contrast, by deploying a mobile UWSN, the investigation team can control the ROV remotely. The self-reconfigurable UWSN tolerates more faults than the existing tethered solution. After investigation, the underwater sensors can be recovered by issuing a command to trigger air-bladder devices.

- Disaster prevention: Sensor networks that measure seismic activity from remote locations can provide tsunami warnings to coastal areas, or study the effects of submarine earthquakes (seaquakes).

- Assisted navigation: Sensors can be used to identify hazards on the seabed, locate dangerous rocks or shoals in shallow waters, mooring positions, submerged wrecks, and to perform bathymetry profiling.

- Distributed tactical surveillance: Autonomous underwater Vehicles (AUVs) and fixed underwater sensors can collaboratively monitor areas for surveillance, reconnaissance, targeting and intrusion detection systems.

Rest of the paper is organized as follows. In section 2, we introduce the communication architecture of underwater acoustic sensor networks. In section 3, we highlight the open research issues of each layer of the underwater acoustic sensor network. Conclusions are given in section 4 .

\section{UNDERWATER ACOUSTIC SENSOR NETWORK}

In this section, we discuss about the underwater network environment, supporting architectures and components.

\section{A. Underwater network environment}

A sample underwater (uw) network environment is as shown in the Fig. 1. The network consists of a set of underwater local area networks (UW-LAN, also known as clusters or cells). Each sensor is connected to the sink within the cluster. The sensors can be connected to uw-sinks via direct paths at multiple hops. The information from the sink of each cluster is transferred to surface station through vertical links. The station at the surface is equipped with acoustic transceivers that are capable of handling multiple parallel communications with the deployed uw-sinks.

\section{A. Underwater network architecture}

The underwater sensor network topology is still an open research issue for the research community. Some of the architectures supporting underwater sensor networks are static two-dimensional under water acoustic sensor networks (UW-ASNs), static three dimensional under water acoustic 


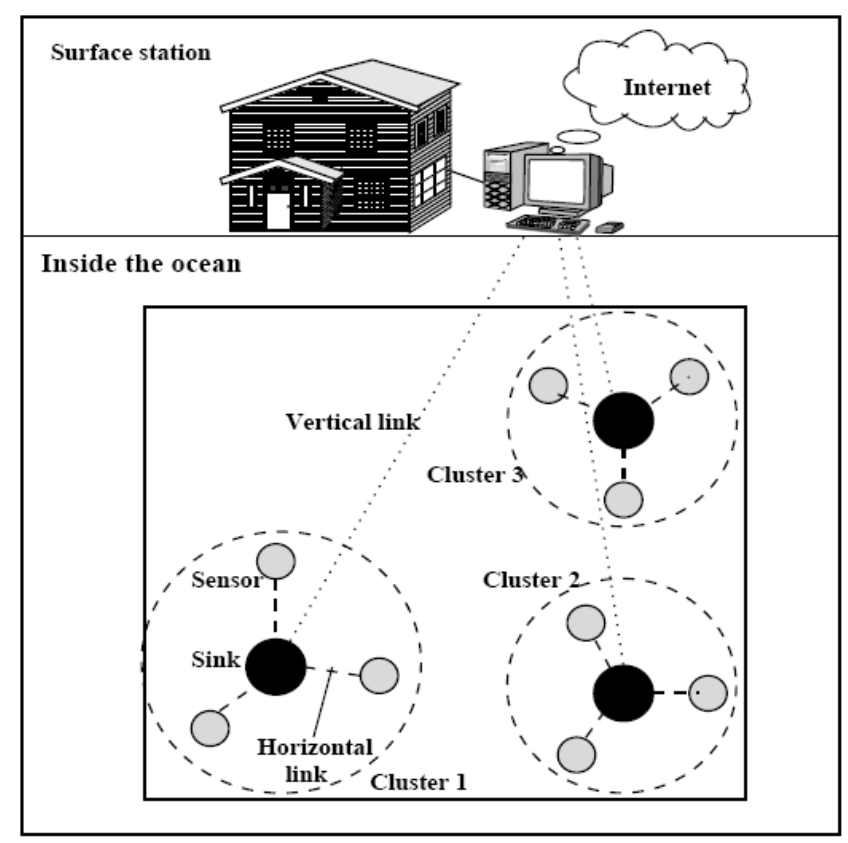

Fig. 1. Underwater Network Environment

sensor networks and three dimensional networks of autonomous underwater vehicles [12].

- Static two-dimensional UW-ASNs: These are constituted by sensor nodes that are anchored to the bottom of the ocean. Typical applications may be environmental monitoring, or monitoring of underwater plates in tectonics.

- Static three-dimensional UW-ASNs: These include networks of sensors and may be used for surveillance applications or monitoring of ocean phenomena (ocean bio-geochemical processes, water streams, pollution).

- Three-dimensional networks of autonomous underwater vehicles (AUVs): These networks include fixed portions composed of anchored sensors and mobile portions constituted by autonomous vehicles.

\section{B. Underwater sensor network components}

Underwater sensor network consists of group of sensor nodes anchored to the bottom of ocean with deep ocean anchors which are in turn connected to one or more underwater gateways (devices in charge of relaying data from ocean bottom to surface station) by means of wireless acoustic links. Sensor nodes float at different depths to observe a given phenomenon. The technique employed for thr purpose are as follows.

1. Each underwater sensor node is attached to a surface buoy by means of wires whose length can be regulated so as to adjust the depth of each sensor node. This solution is easy and deployment is quick but the problem encountered is that floating buoys may obstruct ships navigating on the surface. Since these are floating they can be easily detected and deactivated by enemies in military settings. Floating buoys are also vulnerable to weather and tampering or pilfering.

2. To overcome the above problem, a different approach is proposed. Sensors are anchored to the bottom of ocean and equipped with a floating buoy that can be inflated by pump. The buoy pushes the sensor towards the ocean surface. The depth of the sensor is regulated by adjusting the length of wire that connects the sensor to the anchor, by means of an electronically controlled engine that resides on the sensor.

Underwater gateways (uw-gateways) are devices equipped with two acoustic transceivers, namely a vertical and horizontal transceiver. Horizontal transceiver is used to communicate with the sensor nodes to send commands and configuration data to the sensors (uw-gateways to sensors) and to collect monitored data (sensors to uw-gateway). Vertical transceiver is used for communication from uw-gateway to surface station. The surface station is equipped with an acoustic transceiver that is able to handle multiple parallel communications with a long range RF and/or satellite transmitter to communicate with the onshore sink (os-sink) and/or to a surface sink (s-sink).The communication between the sensors and from sensor to uw-gateway can be accomplished by direct link or multi hop paths. Direct link is the simplest way to network sensors but it is not energy efficient solution as the increased distance will reduce transmission power. Hence network throughput reduces. In multi-hop paths, communication is through multiple sensors, hence results in power saving which in turn increases network efficiency, but the complexity of algorithms at network layer and number of overheads increases .Typical applications may be environmental monitoring, or monitoring of underwater plates in tectonics [13].

\section{RESEARCH CHALLENGES IN UNDERWATER SENSOR NETWORK}

An underwater sensor network poses unique characteristics in networking as compared with the terrestrial networks. In this section we highlight the emerging research issues and challenges imposed by the underwater sensor network. Presentation of the research issues in this section is done with respect to the physical layer, data link layer, network layer, transport layer and application layer.

\section{A. Physical layer}

The communication media that can be chosen for underwater sensor networks are radio frequency waves or optical wave or acoustic wave. The main objective of underwater acoustic communication is to overcome performance limitations observed in dispersive channel and also improve bandwidth efficiency. To achieve high bandwidth efficiency the suitable modulation schemes are as follows.

- Frequency shift keying (FSK): In FSK modulation, information bits are used to select the carrier frequencies of the transmitted signal. The receiver compares the measured power at different frequencies to infer what has been sent. Using only energy detector at the receiver, this scheme bypasses the need for channel estimation, and is thus robust to channel variations. Limitation of this method is guard bands are needed to avoid the interference caused by frequency-spreading, and guard interval is inserted between successive symbol transmissions for channel clearing to avoid the interference caused by time-spreading. As a result, the data rate of FSK is very low. Hence advanced mechanisms are needed for this type of modulation to increase the data rate. 
- $\quad$ Frequency hopped (FH) FSK: FSK improves the data rate as it does not need to wait the channel clearing corresponding to the previous symbol transmission on a different frequency. However, due to the bandwidth expansion via frequency hopping, the overall bandwidth efficiency remains low, typically much below 0.5 bits/sec/Hz. So in the bandwidth constrained underwater sensor networks FSK may not be suitable.

- $\quad$ Code division multiple access (CDMA): CDMA is an important emerging technology for underwater acoustic networks. CDMA permits random, overlapping access to shared communication channel. Hence CDMA may be the suitable modulation technique for the underwater sensor networks.

- Spread spectrum: Modulation can be coherent or non-coherent. For a coherent modulation channel estimation and tracking are needed if phase coherent modulation such as phase shift keying (PSK) is employed. For non-coherent DSSS, different spreading codes can be used and the receiver compares the amplitudes of the outputs from different matched filters, with each one matched to one choice of spreading codes. This avoids the need for channel estimation and tracking in underwater sensor networks.

- Single carrier phase-coherent modulation with adaptive channel equalization: One major step towards high rate communication is the direct transmission of phase-coherent modulations, including phase shift keying (PSK) and quadrature amplitude modulation (QAM) [20]. The channel introduces a great deal of inter-symbol interference (ISI) due to multipath propagation. Advanced signal processing at the receiver side in the underwater sensor networks is required to suppress the interference. This process is termed as channel equalization

- Multicarrier modulation: The idea of multicarrier modulation is to divide the available bandwidth into a large number of overlapping sub bands, so that the waveform duration for the symbol at each sub band is long compared to the multipath spread of the channel [15] [16]. Consequently, inter-symbol interference may be neglected in each sub band, greatly simplifying the receiver complexity of channel equalization. Precisely due to this advantage, multicarrier modulation in the form of orthogonal frequency division multiplexing (OFDM) has prevailed in recent broadband wireless radio applications. However, underwater channels entail large Doppler spread which introduces significant interference among OFDM subcarriers. Lacking effective techniques to suppress the intercarrier interference (ICI), early attempts at applying OFDM to underwater environments had a very limited success.

- Multi-input multi-output techniques: A wireless system that employs multiple transmitters and multiple receivers is referred to as a multiple-input multiple-output (MIMO) system. It has been shown that the channel capacity in a scattering-rich environment increases linearly with the numbers of transmitters and receivers [17] [18]. Hence, MIMO modulation is a promising technology to offer high data rate for underwater acoustic communication.

Open Research issues at physical layer as discussed in [12].

- It is necessary to design and develop inexpensive transmitter/receiver modems for underwater communications.

- Low complexity sub-optimal filters characterized by rapid convergence must be designed.

- It is also necessary to enable real time underwater communications with decreased energy expenditure.

\section{B. Data Link Layer}

There is no need for MAC protocols in existing small scale acoustic networks as the sensors are sparsely separated and point to point communication is sufficient. But the applications requiring dense deployment of sensors in UWSN scenario needs design of an efficient medium access protocol to control communication among users.

Most existing MAC protocols are radio based and assume that signal propagation delay between nodes is negligible but propagation delay is more in underwater networks. The propagation delay of sound in water is five times more than that of radio in air and also bandwidth capacities of acoustic channels are very low compared to RF channels. Hence the MAC protocols for underwater networks should consider long propagation delay, low available bandwidth, energy efficiency and node mobility. Some of the protocols are as follows.

- Frequency Division Multiple Access (FDMA): is a channelized MAC protocol which divides the available bandwidth into several sub-bands and assigns one of the band to a particular user. The band is used by an assigned user only till it is released. FDMA is found suitable for ground based networks but this does not hold good for underwater network for the reasons like:

1. Limited bandwidth, if band is divided into more number of smaller sub bands, the coherence bandwidth of channel may become larger than available FDMA sub-channel

2. Fading occurs among users because of different sub bands

3. Fails during busty traffic as bandwidth will be fixed for each user and cannot be changed [22]-[26].

- Time Division Multiple Access (TDMA) : is the other way of accessing medium based on time slots i.e. time division multiple access technology. In this, the time frame is divided into multiple slots and each slot is assigned to one particular user. TDMA is advantageous in two ways it is energy saving and is flexible. Energy saving is done as user transits information only in allotted slots. It is said to be flexible in underwater sensor networks, because no extra hardware is needed. But the usage of this scheme in underwater sensor networks has some limitations, which are as follows:

1 To avoid collisions from neighbor slots they need to be provided with guard time that are proportional to propagation delay. Hence the overhead increases. It 
is found that overhead induced by TDMA is larger than FDMA [24].

2 It is very difficult to achieve time synchronization which is a must in underwater scenario due to difference in propagation delays.

3 Throughput reduces if time synchronization is not maintained due to collisions.

- Code Division Multiple Access (CDMA): This is based on spread spectrum. CDMA is robust to frequency selective fading caused by multipath since this distinguishes among signals simultaneously which are transmitted by multiple devices through codes. Hence this allows exploiting time diversity in underwater acoustic channels by leveraging Rake filters [27] at receiver to compensate for the effect of multipath. Hence CDMA increases channel reuse and reduces packet retransmissions which increases throughput and battery energy. Here the distinction among users is based on spreading code which is orthogonal with any other spreading codes that other users take. CDMA is a promising technology for most of networks. However it is studied that multi-user CDMA system needs good power control strategy for efficient performance [28]. Otherwise, near far problem might deteriorate its performance [29]. Power control strategies need to be implemented to reduce interference problem which is difficult and challenging especially in underwater scenario.

Dynamic contention-based MAC in a distributed topology is the simpler contention-based distributed protocol that includes half duplex ALOHA, carrier sense multiple access (CSMA) and medium access collision avoidance (MACA) using RTS/CTS handshaking [31]. A CSMA based contention protocol is described in [32]. CSMA prevents collisions with the ongoing transmission at the transmitter side; on receiver side collisions can be avoided by adding a guard time between transmissions which increase propagation delay in the network. Hence the protocol is inefficient for underwater networks. The use of contention based method relies on handshaking signal such as RTS/CTS in shared medium access. But this handshaking is impractical because of the reasons like higher propagation delay for underwater networks, so large delays will be observed in propagation of RTS/CTS, DATA, ACK and so on, hence throughput reduces. Because of large propagation delay, there is a possibility of channel be sensed idle while transmission is in process as data is not being received. Start and finish time of transmissions will be difficult to predict because of high variability of delay in handshaking. Hence number of collisions increases.

- Floor acquisition multiple accesses (FAMA) uses carrier sensing and puts restrictions on RTS/CTS time durations. This is a family of protocols of which MACA is a variant. FAMA protocols permit a station to acquire control of the channel dynamically before transmitting data packets. The floor acquisition strategy is based primarily on a request response (RTS-CTS) control dialogue between a sender and an intended proxy receiver. In addition, carrier sensing is used to increase substantially channel throughput. FAMA with slotting can be used for underwater networks.

- Distance aware collision avoidance Protocol(DACAP): is also based on MACA. But it adds warning message if RTS is overheard while waiting for a reply to its own RTS. While waiting for reply, if another CTS or warning is heard, a random back off is used. Optimal power control for DACAP is studied in [33]. The optimal power is found to be the one that minimizes connectivity. A MAC solution is being introduced for underwater networks with AUVs [30]. The scheme organizes the network into multiple clusters, each composed of adjacent vehicles. Inside the cluster TDMA is being used with large guard bands, to overcome effect of delay. Since the nodes and vehicles are assumed to be close, negative effect of TDMA i.e. high propagation delay and loss caused by guard bands can be limited. Interference among clusters can be minimized by assigning different spreading codes to different clusters.

A deep-water acoustic local area network (ALAN) deployed in Monterey Canyon is presented in [22] [34]. The sub-surface nodes send asynchronous requests via a shared channel (similar to MACA RTS) to send data packets to the master node. The master node sends an acknowledgement (similar to MACA CTS) via a different channel indicating the data channel to be used. The node transmits data immediately on the data channel. The request, ACK, data channels are in different frequency bands. This protocol resembles MACA except that the RTS, CTS and DATA are sent on different channels. Since the transmit request happens on a shared contention channel, it is classified as a contention protocol. MAC topology is centralized as the MAC function of data channel allocation is performed by the central node.

The polling-based protocol called FAMA-CF in [35] uses request for RTS (RRTS), RTS, CTS, DATA, ACK handshaking to communicate with the central node. One CDMA code per cluster and spatial re-use of codes is done in [36]. TDMA is used within each cluster. Nodes are assumed to be able to handle multiple CDMA codes simultaneously. Similar scheme in which clusters are allocated either usage of different CDMA codes or FDMA bands is explained in [37], where within each cluster TDMA is used. Since cluster heads are tasked with TDMA slot allocation to ordinary nodes, we can classify the above as centralized MAC topology. An underwater acoustic cellular network is an extension of centralized topology. Analysis of frequency re-use between adjacent clusters and optimal cell-radius selection criteria has been carried out recently [38]. Channel allocation and scheduling protocol for cellular networks are presented in [39].

Second functionality of data link layer is to provide error control functions for data being transmitted. The properties of channel i.e. path loss and multipath fading leads to high bit error rates. Bit error rate can be monitored by using ARQ, i.e. Automatic repeat request scheme, but this method is not suitable as it indulges high energy cost, latency and overhead increases due to retransmission. Other way is to provide forward error correction (FEC) techniques. In this, redundant information bits are appended purposely so that receiver can detect and correct errors. This helps in overcoming retransmissions but at the same time encoding and decoding schemes have to be implemented. Hence this scheme poses challenge in using processing power efficiently. Also the bandwidth utilization might increase because of redundant bits being added, hence bandwidth efficiency can be 
improved by choosing optimal amount of redundant bits. Major aspect in DLL/MAC is energy conservation as discussed in [22].

A power control and sleep-wake up scheme is proposed in [40] which discusses power controlled and loosely synchronized (PCLS) MAC protocol for tactical underwater surveillance system (TUSS). These protocols reduce the energy consumption and the link layer delay, and also make PCLS resilient against topology changes due to node failures and mobility. Another example on energy minimization [41] shows an ultra-low duty cycle MAC protocol focusing on energy conservation at low data rate. A sensor wakeup scheme - adaptive wakeup schedule function (AWSF), suitable for underwater sensor systems uses a time cyclic wakeup schedule for each node such that at any one time only a few nodes are active [42].

Open research issues at the data link layer are as follows.

- In case CDMA is adopted, it is necessary to design access codes with high auto-correlation and low cross-correlation properties to achieve minimum interference among users. This needs to be achieved even when the transmitting and receiving nodes are not synchronized.

- Research on optimal data packet length is needed to maximize the network efficiency.

- It is necessary to design low-complexity encoders and decoders to limit the processing power required for forward error correction (FEC) functionalities.

- Researchers should evaluate the feasibility and the energy-efficiency of nonconvolutional error control coding schemes.

- Distributed protocols should be devised to reduce the activity of a device when its battery is depleting without compromising on network availability.

Existing MAC protocols for wireless networks are Serial Transmission MAC Protocols (STMP) and Parallel transmission MAC protocols (PTMP) [20] [21]. These protocols may be suitable for underwater sensor networks. STMP statistically multiplexes traffic over a single channel and at any time, the channel can transmit a packet of only one node. It is also called a Single Channel MAC protocol. PTMP divides available bandwidth into several parts and data can be transmitted on each one in parallel. It is also termed as Multi-Channel MAC protocol.

\section{Network Layer}

The function of network layer is to find path from source to the destination taking into account many characteristics of the channel such as long propagation delay, energy of the nodes. There has been intensive study to find the route from source to the destination in different gateways of underwater sensor networks. Routing protocols for ad hoc wireless sensor networks and sensor networks are discussed in [44] [45]. But because of the quite different nature of underwater environment there are several difficulties that need to be addressed for existing underwater networks.

Existing routing protocols are divided into three categories: proactive, reactive and geographical routing. The two main reasons for avoiding proactive protocols in sensor networks in underwater sensor networks are memory and energy [46] [47]. Reactive protocols are unsuitable for underwater networks because of high latency, asymmetrical links and topology. Geographical Routing Protocols work on localization information [48] [49]. The source node selects its next hop based on position of its neighbors and the destination. Lot of research scope is available in this area as it is difficult to find exact localized information in underwater environment with limited energy expenditure. Strict synchronization is needed which is difficult to obtain due to the variable propagation delay of underwater networks. To estimate the geographical location GPS being used in terrestrial cannot be used in underwater. Hence devising new schemes for routing in underwater sensor networks is a challenging issue.

Reference [19] proposes a cluster-based on demand multicast routing protocol (SC-ODMRP) as an extension to the flat multicast routing protocols in large scale ad hoc networks using clustering concept on ODMRP to improve network performance in terms of end-to-end delay and control packets. The paper also proposes a link stability approach to design a stable multicast algorithm. This approach increases data delivery and decreases overhead

Open research issues at the network layer are as follows [10]

- Algorithms and protocols for routing layer need to be redeveloped to provide strict or loose latency bounds for time critical applications, to detect and deal with disconnections caused by failures, unforeseen mobility of nodes or battery depletion.

- There is a need to develop mechanisms to handle loss of connectivity without provoking immediate retransmissions for delay tolerant applications.

- Algorithms must be robust as the quality of link is highly unpredictable.

- Accurate modeling is needed for good understanding of dynamics in data transmission.

- Simulation models and tools need to be developed.

- Local route optimization algorithms are needed to react to consistent variations in metrics describing energy efficiency.

- Mechanisms are needed to integrate AUVs in underwater networks and to enable communication between sensors and AUVs. In particular, all the information available to sophisticated AUVs (trajectory, localization) could be exploited to minimize the signaling needed for reconfigurations.

- In case of geographical routing protocols, it is necessary to devise efficient underwater positioning systems.

\section{Transport Layer}

The function of transport layer in any kind of the network is to provide reliable connectivity from end to end, congestion and flow control. The transport layer of underwater sensor network is totally unexplored area. Existing transport protocols for wireless networks always focus on energy efficient networking solutions with always on connectivity 
between communication end points and short link delays. But these assumptions cannot be applied for underwater networks because of long propagation delay. Conventional data transfer protocol becomes inefficient as they employ idle waiting time. The main objective of transport layer protocol in wireless network is to save scarce resource and improve the efficiency.

Existing transmission control protocol (TCP) implementations are not suitable for underwater networks as the flow control is based on window mechanism which needs accurate round trip time (RTT). The round trip time is measured as stochastic model with high mean and high variance which affects the throughput of the system. Because of high delay, network may drop the packets from queues, hence it is necessary to distinguish between packet loss due to bit error rate or due to drop from queue. TCP protocol assumes that packet loss is due to congestion only and hence reduces the rate of flow of packets in network. But the underwater networks must consider losses due to impairments in channel. Protocols for underwater sensor networks must be framed in such a way that if congestion is the cause of packet loss, transmission rate should be decreased while in cases where loss is due to bad channel quality transmission rate should not be decreased to maintain efficiency. Hence new schemes are to be designed for underwater sensor network.

Event to sink reliable transport (ESRT) protocol is designed for terrestrial network to achieve reliable event detection with minimum energy expenditure [50]. ESRT mechanism relies on spatial correlation among event flows but in underwater sensor network sensors are more sparsely deployed, hence correlation among the sensor readings may not be significant. A staged transport protocol, aDapTN for advanced wireless sensor network is proposed in [51]. This adopts a store-and-forward transport paradigm with an asynchronous wakeup scheme. The transport layer protocol seeks to minimize network idle energy expenditure without compromising end to end data reliability. The transport protocol aDapTN works even when network is partitioned due to scheduled node sleep and unexpected disconnection. Idle energy can be saved by dropping assumption of end to end connectivity between source and destination. Approach for formulation of protocols is based on Delay/Disruption Tolerant Network (DTN) architecture [52] and consists of two key components: DTN-like store-and-forward transport paradigm for data transmission, and an asynchronous wakeup scheme for node rendezvous. Transport layer functionalities can be tightly integrated with data link layer functionalities in a cross-layer module.

A complete transport layer solution for the underwater environment should fulfill the following design principles:

- Shadow zones: Although correct handling of shadow zones requires assistance from the routing layer, a transport protocol should also handle the shadow zones.

- Minimum energy consumption: A transport protocol should be explicitly designed to minimize the energy consumption.

- Rate-based transmission of packets: A transport protocol should be based on rate based transmission of data units as it allows nodes flexible control over the rates.

- Out-of-sequence packet forwarding: Packets should be continuously forwarded to accelerate the packet delivery process.

- Timely reaction to local congestion: A transport protocol should adapt to local conditions immediately, to decrease the response time in case of congestion. Thus, rather than sinks, intermediate nodes should be capable of determining and reacting to local congestion.

- Cross-layer-interaction-based protocol operation: Losses of connectivity or partial packet losses (i.e., bit or packet errors) should trigger the protocol to take appropriate actions. Therefore, unlike in the layered communications paradigm, transport protocol operations and critical decisions should be supported by the available information from lower layers.

- Reliability: A hop-by-hop reliability mechanism surfaces as a prevalent solution as it provides energy efficient communication. However, there should also be mechanism to guarantee the end-to-end reliability.

- SACK-based loss recovery: Many feedbacks with ACK mechanisms would throttle down the utilization of the bandwidth-limited channel unnecessarily. Thus, the notion of selective acknowledgment (SACK), which helps preserve energy, should be considered for loss scenarios where it is not possible to perform error recovery at lower layers only.

Open research issues for transport layer in the underwater sensor networks are as follows [12].

- New flow control strategies need to be devised in order to tackle the high delay and delay variance of the control messages sent back by the receivers.

- New effective mechanisms tailored to the underwater acoustic channel need to be developed, in order to efficiently infer the cause of packet losses.

- New event transport reliability metric definitions need to be proposed, based on the event model and on the underwater acoustic channel model.

- Optimal update policies for the sensor reporting rate are needed, to prevent congestion and maximize the network throughput efficiency as well as the transport reliability in bandwidth limited underwater networks.

- The effects of multiple event occurrences on the reliability and network performance requirements must be studied, as well as efficient mechanisms to deal with it.

- It is necessary to statistically model loss of connectivity events in order to devise mechanisms,

- To enable delay-tolerant applications tailored to the specific underwater requirements.

- Different functionalities at the data link and transport layer such as channel access, reliability and flow control, should be jointly designed and studied.

- A cross-layer approach is highly recommended to accordingly, optimize these mechanisms and make them adaptable to the variability of the characteristics of the underwater channel.

\section{E. Application Layer}

The function of application layer is to provide a network management protocol that makes hardware and software detail of the lower layers transparent to management applications [20]. The functionalities include identification of communication partners, determining resource availability 


\begin{tabular}{|c|c|c|c|c|}
\hline \multirow{3}{*}{$\begin{array}{l}\text { Security } \\
\text { service }\end{array}$} & \multirow{3}{*}{$\begin{array}{c}\text { Power } \\
\text { Management }\end{array}$} & \multirow{3}{*}{$\begin{array}{l}\text { Time } \\
\text { Sync }\end{array}$} & \multirow{3}{*}{$\begin{array}{l}\text { Localization } \\
\text { service }\end{array}$} & $\begin{array}{l}\text { Application } \\
\text { layer }\end{array}$ \\
\hline & & & & $\begin{array}{l}\text { Network } \\
\text { layer }\end{array}$ \\
\hline & & & & $\begin{array}{l}\text { Data link } \\
\text { layer }\end{array}$ \\
\hline \multicolumn{5}{|c|}{ Physical layer } \\
\hline
\end{tabular}

Fig.2 Cross layer protocol stack in underwater sensor networks

and synchronizing the communications, assign tasks and to advertise events and data.

Application layer protocols for terrestrial sensor networks are Telnet, File Transport Protocol (FTP), and Simple Mail Transfer Protocol (SMTP) [53]. San Diego Supercomputing Center Storage Resource Broker (SRB) [54] [55] is a clientserver middleware that provides a uniform interface for connecting to heterogeneous data resources over a network, and accessing replicated data sets. SRB provides a way to access data sets and resources based on their attributes and/or logical names rather than their names or physical locations.

Not much effort has been made to address the specific needs of the underwater acoustic environment. Instead of designing a complete new set of protocols, one can modify existing protocols of ground-based wireless networks to meet the underwater acoustic network (UAN) needs. Thus, it is necessary to understand the application areas and the communication issues for UANs, and to apply its uniqueness into the existing application protocols.

\section{F. A Cross-layer Protocol Stack}

A protocol stack for uw-sensors should combine power awareness and management, and promote cooperation among the sensor nodes [12]. It should consist of physical layer, data link layer, network layer, transport layer, and application layer functionalities. The protocol stack should also include a power management plane, a coordination plane, and a localization plane (see Fig. 2). The power management plane is responsible for network functionalities aimed at minimizing the energy consumption (e.g., sleep modes, power control). The coordination plane is responsible for all functionalities that require coordination among sensors, (e.g., coordination of the sleep modes, data aggregation, etc.). The localization plane is responsible for providing absolute or relative localization information to the sensor node, when needed by the protocol stack or by the application.

While all the research on underwater networking so far has followed the traditional layered approach for network design, it is an increasingly accepted opinion in the wireless networking community that the improved network efficiency, especially in critical environments, can be obtained with a cross-layer design approach. These techniques will entail a joint design of different network functionalities, from modem design to MAC and routing, from channel coding and modulation to source compression and transport layer, with the objective to overcome the shortcomings of a layered approach that lacks information sharing across protocol layers, forcing the network to operate in a suboptimal mode.

\section{Conclusion}

Underwater networks of sensors have the potential to enable unexplored applications. These potential applications will be made viable by enabling communications among underwater devices. Underwater Acoustic Sensor Networks will consist of sensors and vehicles deployed underwater and networked via acoustic links to perform collaborative monitoring tasks. In this paper, we have presented the basic conceptual architecture of underwater acoustic sensor network. We have discussed the challenges posed by the underwater channel, characteristics of the underwater channel. also the research issues and challenges of each layer in the network protocol stack of underwater sensor network. By the literature survey, we may conclude that research on the underwater sensor network is still in the developing stage.

\section{REFERENCES}

[1] A. Cerpa, J. Elson, D. Estrin, L. Girod, M. Hamilton, and J. Zhao, Habitat monitoring: Application driver for wireless communications technology, In Proceedings of the ACM SIGCOMM Workshop on Data communications in Latin America and the Caribbean, Apr. 2001.

[2] A. Mainwaring, J. Polastre, R. Szewczyk, and D. Culler, Wireless sensor networks for habitat monitoring, In Proceedings of the ACM Workshop on Sensor Networks and Applications, Atlanta, Georgia, USA, Sept. 2002.

[3] D. Whang, N. Xu, S. Rangwala, K. Chintalapudi, R. Govindan, and J. Wallace, Development of an embedded sensing system for structural health monitoring, In Proceedings of the International Workshop on Smart Materials and Structures Technology, Jan. 2004.

[4] N. Ramanathan, M. Yarvis, J. Chhabra, N. Kushalnagar, L. Krishnamurthy, and D. Estrin, A stream-oriented power management protocol for low duty cycle sensor network applications, In Proceedings of the IEEE Workshop on Embedded Networked Sensors, Sydney, Australia, May 2005.

[5] Ocean Engineering at Florida Atlantic University, Available online at: http://www.oe.fau.edu/research/ams.html.

[6] AUV Laboratory at MIT Sea Grant, Available online at: http://auvlab.mit.edu/.

[7] X. Yang, K.G. Ong, W.R. Dreschel, K. Zeng, C.S. Mungle, and C.A. Grimes, Design of a wireless sensor network for long-term, insitu monitoring of an aqueous environment, pp. 455-472, Sensors 2, Vol. $11,2002$.

[8] N.N. Soreide, C.E. Woody, and S.M. Holt, Overview of ocean based buoys and drifters: Present applications and future needs, In proceedings 16th International Conference on Interactive Information and Processing Systems (IIPS) for Meteorology, Oceanography, and Hydrology, Long Beach, CA, USA, January 2004.

[9] L. Freitag, M. Grund, C. V. Alt, R. Stokey and T. Austin, A Shallow Water Acoustic Network for Mine Countermeasures Operations with Autonomous Underwater Vehicles, In Underwater Defense Technology (UDT), 2005. www.hydroidinc.com/pdfs/Acoustic_Network_for_MCM.pdf

[10] J.H. Cui, J. Kong, M. Gerla, and S. Zhou, Challenges: Building scalable mobile underwater wireless sensor networks for aquatic applications, IEEE Network, Special Issue on Wireless Sensor Networking, pp. 12-18, 2006.

[11] Vasilescu.K.Kotay and D.Rus, Data Collection, Storage and Retrieval with an underwater Sensor Network, ACM SenSys'05 pp.154-165, 2005.

[12] I. F. Akyildiz, D. Pompili, and T. Melodia, Underwater acoustic sensor networks: Research challenges, Ad Hoc Networks, pp. 257-279, 2005.

[13] L. Freitag and M. Stojanovic, Acoustic Communications for Regional Undersea Observatories, In Proceedings of Oceanology International, London, UK, Mar. 2002. 
[14] M. Stojanovic, J. A. Catipovic, and J. G. Proakis, Phase-coherent digital communications for underwater acoustic channels, IEEE Journal of Oceanic Engineering, vol. 19, no. 1, pp. 100-111, Jan. 1994.

[15] J. A. C. Bingham, Multicarrier modulation for data transmission: An idea whose time has come, IEEE Communications Magazine, pp. 5-14, May 1990.

[16] Z. Wang and G. B. Giannakis, Wireless multicarrier communications: Where Fourier meets Shannon, IEEE Signal Processing Magazine, vol. 17, no. 3, pp. 29-48, May 2000.

[17] G. J. Foschini and M. J. Gans, On limits of wireless communication in a fading environment when using multiple antennas, WirelessPersonal Communications, vol. 6, no. 3, pp. 311-335, Mar. 1998.

[18] I. E. Telatar, Capacity of multi-antenna Gaussian channels, Bell Laboratories Technical Memorandum, 1995.

[19] S. Roy, T. M. Duman, V. McDonald, and J. G. Proakis, High rate communication for underwater acoustic channels using multiple transmitters and space-time coding: Receiver structures and experimental results, IEEE Journal of Oceanic Engineering, vol. 32, no. 3, pp. 663-688, Jul. 2007.

[20] Zaihan Jiang, Underwater Acoustic Networks - Issues and Solutions, International journal of intelligent control and systems, VOL. 13, NO. 3, SEPTEMBER 2008, 152-161

[21] Z. Jiang, M. Zhou, A prioritized parallel transmission MAC protocol for all-IP wireless WAN beyond $3 G$, Proc of IEEE International Conference on Systems, Man and Cybernetics, 2003, Vol. 4, pp.: 3852-3857, Washing, DC, USA, Oct. 2003.

[22] Mandar Chitre, Shiraz Shahabudeen, Lee Freitag, Milica Stojanovic, Recent Advances in Underwater Acoustic Communications and Networking.

[23] C. Bjerrum-Niese and R. Lutzen, Stochastic simulation of acoustic communication in turbulent shallow water, IEEE journal of Oceanic Engineering, vol. 25, pp. 523-532, 2000.

[24] C. Bjerrum-Niese, L. Bjorno, M. A. Pinto, and B. A. Quellec, $A$ simulation tool for high data-rate acoustic communication in a shallow-water, timevarying channel, IEEE Journal of Oceanic Engineering, vol. 21, pp. 143-149, 1996.

[25] M. Chitre,A high-frequency warm shallow water acoustic communications channel model and measurements, The Journal of the Acoustical Society of America, vol. 122, pp. 2580-2586, 2007.

[26] M. Badiey, B. G. Katsnelson, J. F. Lynch, and S. Pereselkov, Frequency dependence and intensity fluctuations due to shallow water internal waves, The Journal of the Acoustical Society of America, vol. 122, pp. 747-760, 2007.

[27] E. Sozer, J. Proakis, M. Stojanovic, J. Rice, A. Benson, and M. Hatch, Direct Sequence Spread Spectrum based Modem for Underwater Acoustic Communication and Channel Measurements, In Proceedings of MTS/IEEE OCEANS. Nov. 1999.

[28] T. S. Rappaport, "Wireless Communications", Englewood Cliffs, NJ: Prentice Hall, 1996.

[29] M. Stojanovic and Z. Zvonar, Multichannel Processing of Broad-Band Multiuser Communication Signals in Shallow Water Acoustic Channels, IEEE Journal of Oceanic Engineering, 21(2):156-166, Apr. 1996

[30] F. Salva-Garau and M. Stojanovic, Multi-cluster Protocol for Ad Hoc Mobile Underwater Acoustic Networks, In Proceedings of MTS/IEEE OCEANS. San Francisco, CA, Sep. 2003.

[31] E. M. Sozer, M. Stojanovic, and J. G. Proakis, Underwater acoustic networks, IEEE Journal of Oceanic Engineering, vol. 25, pp. 72-83, 2000.

[32] M. Smith, J. C. Park, and A. Neel, A peer-to-peer communication protocol for underwater acoustic communication, In Proceedings of MTS/IEEE OCEANS '97, 1997, pp. 268-272 vol.1.

[33] A. P. Dolc and M. Stojanovic, Optimizing the Transmission Range in an Acoustic Underwater Network, In OCEANS'07, Vancouver, Canada, 2007.

[34] Brady and J. Catipovicl Adaptive multiuser detection for underwater acoustical channels,IEEE Journal of Oceanic Engineering, vol. 19, pp. 158-165, 1994

[35] A. Kebkal, K. Kebkal, and M. Komar,Data-link protocol for underwater acoustic network, In Oceans pp. 1174-1180 Vol. 2, 2005

[36] F. Salva-Garau and M. Stojanovic, Multi-cluster protocol for ad hoc mobile underwater acoustic networks, In OCEANS pp. 91-98 Vol.1, 2003.

[37] P. Casari, S. Marella, and M. Zorzi, A Comparison of Multiple Access Techniques in Clustered Underwater Acoustic Networks, In IEEE Oceans' 07, Aberdeen, Scotland, 2007.
[38] M. Stojanovic, Frequency reuse underwater: capacity of an acoustic cellular network, In Proceedings of the second workshop on Underwater networks Montreal, Quebec, Canada: ACM, 2007.

[39] B. Peleato and M. Stojanovic, A Channel Sharing Scheme for Underwater Cellular Networks, in IEEE Oceans' 07, Aberdeen, Scotland, 2007.

[40] K. Turgay and C. Erdal, A Mac Protocol for Tactical Underwater Surveillance Networks, In Military Communications Conference,pp. 1-7, 2006.

[41] V. Rodoplu and P. Min Kyoung, An energyefficient MAC protocol for underwater wireless acoustic networks, In MTS/IEEE OCEANS, pp. 1198-1203 Vol. 2, 2005.

[42] Y. F. Wong, L. H. Ngoh, W. C. Wong, and W. K. G. Seah, Intelligent Sensor Monitoring For Industrial Underwater Applications, In IEEE International Conference on Informatics, pp. 144-149, 2006.

[43] M. Chitre and M. Motani, On the use of rate-less codes in underwater acoustic file transfers, In OCEANS, pp. 1-6, 2007.

[44] Abolhasan M., et. al., A Review of Routing Protocols for Mobile Ad Hoc Networks, Ad Hoc Networks (Elsevier), vol. 2, pp. 1-22, Jan. 2004.

[45] Akkaya K. and Younis M., A Survey on Routing Protocols for Wireless Sensor Networks, Ad Hoc Networks (Elsevier), vol. 3, pp. 325-349, May 2005.

[46] Jacquet P., Muthlethaler, P., et. al., Optimized Link State Routing Protocol for Ad Hoc Networks, In Proceedings of IEEE Multi Topic Conference (INMIC), (Pakistan), pp. 62-68, Dec. 2001.

[47] Perkins, C. and Bhagwat, P., Highly Dynamic Destination Sequenced Distance Vector Routing (DSDV) for Mobile Computer In Proceedings of ACM Special Interest Group on Data Communications (SIGCOMM), (London, UK), 1994.

[48] Bose, P., Morin, P., et. al., Routing with Guaranteed Delivery in Ad Hoc Wireless Networks, ACM-Kluver Wireless Networks, Springer, vol. 7, pp. 609-616, Nov. 2001.

[49] Melodia, T., et. al., Optimal Local Topology Knowledge for Energy Efficient Geographical Routing in Sensor Networks, In Proceedings of IEEE Conference on Computer Communications (INFOCOM), Hong Kong, Mar. 2004.

[50] O.B. Akan, I.F. Akyildiz, Event-to-sink reliable transport in wireless sensor networks, IEEE/ACM Transactions on Networking.

[51] Yong Wang, ChiehYih Wany, Margaret Martonosi, LiShiuan Peh Transport Layer Approaches for Improving Idle Energy in Challenged Sensor Networks, SIGCOMM'06 Workshops, September 11 15, 2006.

[52] K. Fall. A delay-tolerant network architecture for challenged Internets, In Proceedings of ACM SIGCOMM, 2003.

[53] http://en.wikipedia.org/wiki/OSI_model_Layer_7_Application_ layer, September, 2008.

[54] SDSC Storage Resource Broker, Available from <http:// www.npaci.edu/DICE/SRB/.

[55] C. Baru, R. Moore, A. Rajasekar, M. Wan, The SDSC storage resource broker, In Proceedings of the Centre for Advanced Studies on Collaborative Research, 1998.

[56] Lanbo Liu, Shengli Zhou, and Jun-Hong Cui Prospects and Problems of Wireless Communication for Underwater Sensor Networks, WILEY WCMC SPECIAL ISSUE ON UNDERWATER SENSOR NETWORKS (INVITED),pp.977-994 July 2008

Manjula.R.B. has completed her B.E (ECE) and M.Tech (Digital Communication) from Visvesvaraya Technological University (VTU), Belgaum, India. Currently she is working as faculty in Reva Institute of Technology, Bangalore, India. She has some National/International Conference publications to her credit. Her areas of interest include wireless communication, underwater acoustic sensor networks. She is a Member ISTE (India)

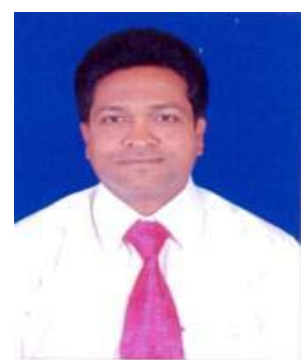

Sunilkumar S. Manvi has obtained his $\mathrm{PhD}$ from Indian Institute of Science (IISc), Bangalore in 2004. To his credit, he has authored 3 books, about 40 National/International Journal publications and about 100 National/International Conference publications. He is guiding $7 \mathrm{PhD}$ students. Currently he is working as Professor and Head, Department of ECE, Reva Institute of Technology and Management, Bangalore, India. His areas of interest include agent technology, grid computing, vehicular ad hoc networks, wireless sensor and ad hoc networks, e-commerce and U-commerce. etc. he is member of IEEE. 